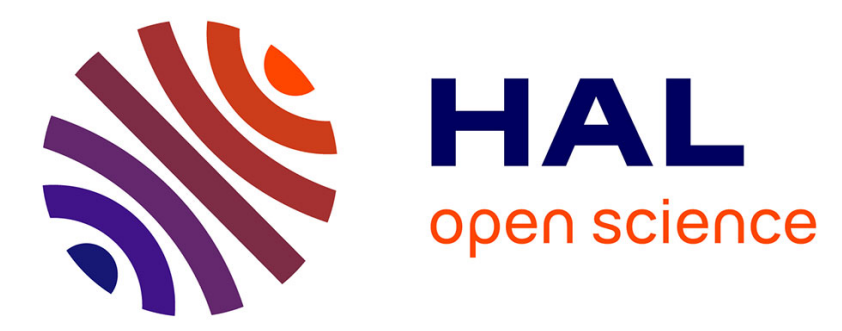

\title{
How much the wavelength dimensioning methods and a tightened QoS provision impact on the dynamic WDM optical networks capacity? (NESIM Best Research Award)
}

Nicolas Jara, Hermann Pempelfort, Gerardo Rubino, Reinaldo Vallejos

\section{To cite this version:}

Nicolas Jara, Hermann Pempelfort, Gerardo Rubino, Reinaldo Vallejos. How much the wavelength dimensioning methods and a tightened QoS provision impact on the dynamic WDM optical networks capacity? (NESIM Best Research Award). Optical Switching and Networking, In press, 35, pp.1-12. 10.1016/j.osn.2019.100540 . hal-02431514

\author{
HAL Id: hal-02431514 \\ https://hal.inria.fr/hal-02431514
}

Submitted on 7 Jan 2020

HAL is a multi-disciplinary open access archive for the deposit and dissemination of scientific research documents, whether they are published or not. The documents may come from teaching and research institutions in France or abroad, or from public or private research centers.
L'archive ouverte pluridisciplinaire HAL, est destinée au dépôt et à la diffusion de documents scientifiques de niveau recherche, publiés ou non, émanant des établissements d'enseignement et de recherche français ou étrangers, des laboratoires publics ou privés. 


\title{
How much the wavelength dimensioning methods and a tightened QoS provision impact on the dynamic WDM optical networks capacity?
}

\author{
Nicolás Jara $^{\mathrm{a}}$, Hermann Pempelfort ${ }^{\mathrm{a}}$, Gerardo Rubino ${ }^{\mathrm{b}}$, Reinaldo Vallejos ${ }^{\mathrm{a}}$ \\ ${ }^{a}$ UTFSM, Valparaiso, Chile \\ ${ }^{b}$ INRIA Rennes - Bretagne Atlantique, Rennes
}

\begin{abstract}
This article gives an insight on the importance to assign a different capacity to each network link and to tighten the Quality of Service (QoS) provision in the network, in dynamic WDM Optical Networks with wavelength continuity constraints. In the text, several examples illustrate the performance of different strategies.

The usual procedure to compute the number of wavelengths of each network link consists of choosing the same capacity to each link on the network. This decision is selected, in most cases, by simplicity and simulation limitations. Notwithstanding, there are no technical motives to force a uniform capacity on the network. In consequence, we analyze the impact on the network capacity of designating the minimum amount of wavelengths to each network link, while satisfying each user QoS requirement.

Moreover, despite the chosen dimensioning strategy, it is usual to offer a much better QoS than requested for some users, with the corresponding waste of resources. We can amend this issue by limiting which wavelengths are available to each user according to its particular QoS constraint. This technique, known as Wavelength Grouping, seeks to provide to each user a QoS level closer to the one defined in the Service Level Agreement. By doing so, it is possible to reduce the network capacity needed to allow communication to its user, or diminishing the network blocking probability for a given wavelength capacity.

In spite of the usual approach, in this work, we demonstrate that a nonuniform dimensioning strategy and a tighten QoS provision allows to save significant networks capacity, while simultaneously provisioning to each user the QoS established in its Service Level Agreement. This fact is very relevant nowadays, where an impending capacity crunch in optical networks is an important issue.
\end{abstract}

Keywords: Optical Networks, Blocking Probability, Wavelength

Email addresses: nicolas.jara@usm.cl (Nicolás Jara), hermann.pempelfort@usm.cl (Hermann Pempelfort), gerardo.rubino@inria.fr (Gerardo Rubino), reinaldo.vallejos@usm.cl (Reinaldo Vallejos)

Preprint submitted to Journal of Optical Switching and Networking 
Dimensioning, Capacity Crunch, Quality of Service.

\section{Introduction}

This decade, researchers anticipated a potential Capacity Crunch problem associated with the increasing traffic demands to telecommunication networks, especially over optical systems 1, 2, 3. This problem refers to the fact that

5 the transmission capacity of an optical fiber is not limitless. Nonetheless, the bandwidth demand has increased exponentially in the last years, and it seems that this phenomenon will continue in the years to come.

There are two possible solutions to this problem. First, to increase the resources installed by multiplying the fiber cables and equipment. However,

10 this option leads to considerable investments in the network deployed. Second, efficiently use the already installed optical systems, since current networks operation is inefficient in the usage of network resources [2, 4]. Seemingly, it is inevitable to face both solutions strategies. However, the second one can be implemented in the short term. Therefore, our work is focused on efficiently manage the resource available on current optical networks.

Nowadays optical networks are inefficient since (among other issues) they are operated statically 5 . This operation allocates network resources to each user permanently from source to destination, regardless of the percentage of time it is used. However, to overcome the said problem, it is possible to migrate 20 current networks operation from a static to a dynamic operation [6. The latter is denoted as Dynamic Optical Networks, consisting in allocating the resources to a given user only when it requests communication. Although it is already possible to deploy this type of technology, this has not happened yet because it is unclear how much network capacity savings can be expected compared to 25 the necessary investments 7 .

To go into details, a central issue in the design of dynamic optical networks is to compute the number of wavelengths to each network link, known as Wavelength Dimensioning (WD) 8 problem. This measure is important because the cost of many components in an optical network is strongly affected by this

30 parameter. In fact, it determines how many infrastructure resources are needed on the network to achieve the network operation. To achieve an efficient wavelength dimensioning on dynamic networks, two contradictory objectives must be satisfied: first, to diminish the network capacity; and second, to ensure a given level of quality of service to each users, measured by the user's blocking 35 probability. In other words, the network is designed to offer to the users a very low blocking probability while saving significant network resources. This strategy is the same one used, prior Internet arises, in telephone networks planning methods.

Most wavelength dimensioning strategies consider a worst-case scenario to 40 compute the capacity of the links in optical networks. This scheme, denoted as Uniform Dimensioning (UD), assigns the same number of wavelengths to each link, while ensuring a given quality of service (QoS) to all users, even on the 
more congested link [9, 10, 11. However, it is quite common to find network links with higher usage than others, measured by the number of users, or the 45 traffic load on the links (for instance, the commonly used shortest path strategy tends to concentrate the majority of the user paths on a subset of links of the network). This uneven situation suggests that it is not necessary to assign the same capacity to all the links on the network, and in this way, saving network resources while providing the same services. The latter strategy is called as ${ }_{50}$ Non-Uniform Dimensioning (NUD). Let us exemplify how the NUD strategy may work. Consider two links of a network, with 5 and 4 wavelengths on the first and second links, respectively. The frequencies used on each wavelength are denoted as $\lambda_{1}, \ldots, \lambda_{5}$ on the first link, and $\lambda_{1}, \ldots, \lambda_{4}$ on the second one. The first four wavelengths are the same on both links, thus to allow communication 5 using both links, the users can only use simultaneously one of the first four wavelengths when available. On the other hand, the 5th wavelength on the first link can only be used by users with only the first link among their paths, not the second one. Therefore, in this work, we compute the number of wavelengths of each network link, to assess the impact of both approaches on the network 60 capacity, while ensuring a minimum acceptable QoS to each user.

The dimensioning problem on dynamic WDM optical networks is specially tricky when the network has wavelength continuity constraint, (the case analyzed here). This constraint means that when a user wants to transmit, the same wavelength has to be available on every link belonging to the given user route

65 (end-to-end). However, the chosen wavelength may change over time. This situation means that every time the user request resources to send data, a procedure searches an available wavelength on the user route links, with the wavelength continuity restriction. The search for an available wavelength is another important problem in dynamic optical networks planning, called the "Wavelength Assignment" ( $W A$ ) problem [8, 12].

The wavelength assignment problem has been widely covered in the literature. Some of the most common heuristics proposed are First-Fit (FF), Random Fit (RF), Most-Used (MU), and Least-Used (LU), among others [8, 12, 13, 11. First-Fit is the most popular procedure in the literature since it performs better 75 in terms of blocking probabilities, with low complexity. As a consequence, on our research we use this procedure to allocate the wavelengths.

Another long-standing issue in optical network planning is to ensure an endto-end quality level for all users. The Service Level Agreement (SLA), signed by the service providers and their clients, defines the minimum quality of serso vice (QoS) acceptable for each user, measured as a probabilistic guarantee. The definition of these bounds is obtained considering objective criteria, such as: different quality of service requirements [14, 15, 16]; and subjective decisions, such as network scalability requirements. Based on these QoS agreements, engineers must design the network fulfilling said QoS requirements. For instance, 85 despite the dimensioning strategy, the capacity computed to each link has to guarantee each user QoS constraint.

Seemingly, the Non-Uniform dimensioning strategy is more efficient in taking care of resources, but it is necessary to assess how significant it is. This 
efficiency may be important, since using Uniform dimensioning strategies may dimension more capacity than necessary, which is unacceptable in a capacity crunch situation. Then, our first goal is to assess the impact of using a Uniform or Non-Uniform dimensioning technique on the network capacity.

Once the dimensioning strategy is decided, a topic that arises is how to ensure a given QoS level required by each user. The common approach is to

95 satisfy the QoS to all users [8, 12, 9, 11, 17, guaranteeing the quality of service of the most demanding one, which reduces to give all users the same worst-case scenario QoS, obviously wasting resources. However, again with an impending capacity crunch approaching optical networks, this over-provision of QoS to some users may achieve significant waste of resource. This situation has been taking care off on a different context. For example, in the OBS context, diverse schemes have been presented concerning the quality of services, such as Wavelength Grouping and Intentional Dropping, among others [18, 19, 20]. However, this situation has not been studied in the current optical networks with wavelength continuity constraints. Consequently, our second goal is to evaluate the impact on applying Wavelength Grouping to adjust the closeness of the real QoS offered and the one defined on the SLA.

According to the previous discussion, this paper analyzes the significance of 2 straightforward decisions in network planning on dynamic WDM optical networks with wavelength continuity constraints. These decisions are: to compute 110 a different amount of wavelengths to each network link; and to offer to each user a QoS close to the one required in the corresponding Service Level Agreement (SLA). Remark that any solution must satisfy all users QoS restrictions (SLA).

To the best of our understanding, there is not a study of the impact of these decisions in the context of optical networks with wavelength continuity

constraints, notwithstanding they may achieve meaningful savings in terms of network resources. In fact, the numerical examples in this article highlight that both strategies (Non-Uniform Dimensioning and Tight QoS provision) allows to obtain important savings on the network capacity, while guaranteeing the required QoS to each user, established on the Service Level Agreement. Regard 120 that all the strategies compared here are executed prior to network operation, thus not affecting the network operation complexity.

The remainder of this paper is as follows: In Section 2 we present both strategies to be analyzed. Section 3 contains some numerical examples comparing the QoS provision and wavelength dimensioning mechanisms in a set of different scenarios. Finally, we give some conclusions and remarks in Section 4.

\section{Network Planning}

In this section first, we explain the model, and then the different network planning decisions to be analyzed.

\subsection{Network and Traffic Model}

${ }_{130}$ We represent the network topology by a graph $\mathcal{G}=(\mathcal{N}, \mathcal{L})$, where $\mathcal{N}$ is the set of network nodes or vertices and $\mathcal{L}$ is the set of directional links (the arcs in 
$\mathcal{G}$ ), with respective cardinalities $|\mathcal{N}|=N$ and $|\mathcal{L}|=L$. The set of users $\mathcal{X} \subseteq \mathcal{N}^{2}$, with cardinality $|\mathcal{X}|=X$, is composed by all the source-destination pairs with communication between them.

We rendered the traffic by an ON-OFF model. Consider user $c$ : during any of its ON periods, whose average length is $t_{O N c}$, the source transmits at a constant rate (which is the rate associated with the used technology); meanwhile, during an OFF period, with average length $t_{O F F c}$, the source is silent, accumulating data to be transmitted in the next ON period.

To simplify the presentation, we take as transmission rate unit the used transmission speed. The traffic load of user $c$, denoted by $\varrho_{c}$, is:

$$
\varrho_{c}=\frac{t_{O N_{c}}}{t_{O N_{c}}+t_{O F F c}} \text {. }
$$

of each network link $\ell \in \mathcal{L}$. To do so, we must assign to each link the number of wavelengths necessary to provide communication to its users while satisfying two contradictory objectives. First, to achieve significant savings in network resources and, in the meantime, to ensure some standard end-to-end quality level to all the users in the network. Quality level means, here, maximal user blocking probability.

As mentioned in this paper introduction, most methods to compute the network capacity consider a worst-case scenario, assigning the same number of wavelengths to each network link 9, 10, 11 (Uniform Dimensioning strategy).

65 However, to allocate a different amount of wavelengths to each link may provide benefits in terms of network savings, while still ensuring the agreed level of quality of service to each user. To compare the cost implications of both techniques, we apply here both approaches to calculate the number of wavelengths of each network link.

Despite the strategy chosen to compute the capacity of the links, the management of the wavelength on each link affects the QoS offered to each user. 
The standard strategy is that all user can transmit on any wavelength available on their link paths if the continuity constraint is satisfied. A different approach consists in restricting the wavelengths available to each user. In a nutshell, this approach computes separately how many wavelengths each user can use to transmit on each link despite the capacity of the links while always ensuring the required QoS on the SLA. Then, each user has a different amount of wavelengths available to communicate despite using the same links. We call this Tight Quality of Service policy (TQoS). This policy helps to reduce the number 180 of wavelengths required on the network, while still guaranteeing a specific QoS to each network user.

To ensure a level of quality of service to each user while dimensioning the network resources, we need a method to evaluate the users blocking probability. Even more, this method must support the TQoS policy and any wavelength dimensioning mechanism. One strategy is to compute the blocking probability employing simulation techniques 23, 12, 22, but it is a time-consuming task depending on the scenarios evaluated. On the other hand, exact analytic methods may be faster, but most of the time, hard to achieve. Given the complexity of the exact solution, in 24] we develop a mathematical method (called "LIBPE")

190 to obtain an accurate while light cost approximate computational scheme, to evaluate the blocking probability, considering all essential aspects and assumptions in optical networks. This procedure is called "LIBPE". Note that the more critical issues to the model are the wavelength continuity constraints and the heterogeneous traffic load of Eq. (1), compatible with any wavelength dimen-

195 sioning mechanism, and the TQOS policy. To the best of our knowledge, LIBPE is the sole (analytic) method supporting the TQoS policy. To further details, in Appendix A, we give an explain this method.

Next, we present two pseudo-codes to compute the wavelength dimensioning. One is assigning the same amount of wavelengths to each network link (Uniform 200 Dimensioning or UD), and the other is with a different capacity per link (NonUniform Dimensioning or NUD). In both cases, we use the TQoS policy.

\subsubsection{Uniform Dimensioning method}

Let the wavelengths be numbered sequentially (i.e. $1,2, \ldots W_{\ell}$ ).

The wavelength assignment ( $W A$ ) problem consists in finding, for each user $c \in \mathcal{X}$, the same wavelength available on all the links in its route. Here we use the First-Fit wavelength assignment approach since it is simple and has associated an excellent performance in terms of blocking probability, with a small computational overhead [8, 13, 11, 25]. In a nutshell, the method orders the different wavelengths, and sequentially searches until there is one available on the whole path; if not, the user request is blocked (lost). The First-Fit strategy is a well-known strategy; thus, it will not be outlined in pseudo-code due to its trivial implementation. However, it is mandatory to present the First-Fit technique altered with the Tight QoS provision policy for a clear understanding.

In Figure 1. we describe, in simple algorithmic form, the Uniform Dimen215 sioning method, including the TQoS modification of the First-Fit wavelength 


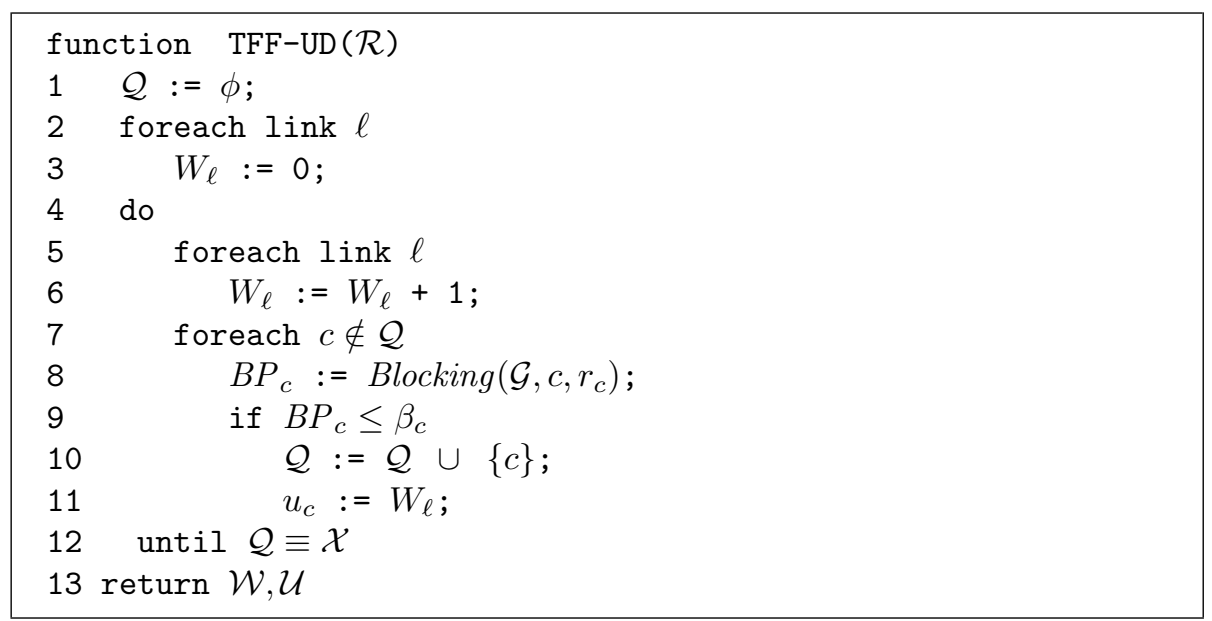

Figure 1: TFF-UD procedure to compute the number of wavelengths on the network and the amount of wavelengths available to each user using a First-Fit wavelength assignment with a tight quality of service policy.

allocation strategy. We call this procedure Tighten First-Fit with Uniform Dimensioning (TFF-UD).

In the pseudo-code given in Figure 1, we include the sub-procedure Blocking evaluating the blocking probability of each user by any means available in the literature. As stated before, in our case, we use the procedure called "LIBPE" [24, 26]. Nevertheless, recall that any available technique can be used to estimate this QoS parameter (for instance, the reader can use a simulation procedure) if it is compatible with the problem restrictions.

The main idea of the procedure displayed in Figure 1 is to increment iteratively the value $W_{\ell}$, on each link $\ell \in \mathcal{L}$, until every network user $c$ satisfies its service level agreement given by the upper-bound $\beta_{c}$. Let us define $\mathcal{Q} \subseteq \mathcal{X}$, as the set of users with their QoS constraint satisfied (maximum acceptable blocking probability). Initially, $\mathcal{Q}$ starts empty, since no user has yet been checked to see if it satisfies its QoS requirement, and each network link $\ell$ has its $W_{\ell}$ value equal to 0 (lines 1 to 3 in Figure 1). The iterative procedure begins inline 4 , and its first step is to increase the number of wavelengths on every link $W_{\ell}$ by 1 . Notice that all the links are augmented together, leading to a uniform wavelength dimensioning.

In the loop starting at line 7 for all user that have not yet satisfy its QoS, in line 8, we evaluate the user $c$ blocking probability $B P_{c}$ by the sub-procedure $\operatorname{Blocking}\left(\mathcal{G}, c, r_{c}\right)$. Then, we check for the same users, if the inequality $B P_{c} \leq \beta_{c}$ is satisfied by the current network capacity $(\mathcal{W})$. Lets define the set $\mathcal{U}=\left\{u_{c} \mid\right.$ $c \in \mathcal{X}\}$ where $u_{c}$ is the maximum available wavelength for user $c$. This means that user $c$ can only use wavelengths $1,2, \ldots, u_{c}$, with $u_{c} \leq \mathcal{W}$. If user $c$ reaches 240 its threshold requirement, then it is included in the $\mathcal{Q}$ set (line 10), and the 
current network capacity $(\mathcal{W})$ is stored as the maximum available wavelength for that user. If some users remain with their QoS requirement unsatisfied, then line 4 to 12 is repeated, until all user's requirements are fulfilled.

The precedent procedure allows providing the Tight Quality of Service policy. This policy means that each user $c$ can use wavelengths $1,2, \ldots, u_{c}$ only. If none of this wavelengths are available in all the links of its path links, then the communication request is rejected (blocked), even if there are more wavelengths available after the $u_{c}$ one (from $u_{c}+1$ to $W_{\ell}$ ). Recall that we want to obtain a small difference between the real QoS obtained and the one required by the

The procedure ends when every link has fulfilled all their users QoS requirements, thus when $\mathcal{Q} \equiv \mathcal{X}$ (line 12). Symbolically, the execution of the whole procedure is written $\{\mathcal{W}, \mathcal{U}\}:=\operatorname{TFF}-\mathrm{UD}(\mathcal{R})$, since its outputs are $\mathcal{W}$, the set of wavelengths, and $\mathcal{U}$, the set of available wavelengths for each user.

\subsubsection{Non-Uniform Dimensioning method}

To implement the TQoS policy with a Non-Uniform wavelength dimensioning, we modify the algorithm displayed in Figure 1 to separately compute the number of wavelengths to each network link. This alteration on the procedure allows calculating a different amount of wavelengths to each link while ensur-

260 ing the corresponding maximum blocking probability to each user, including the TQoS policy. We call this procedure Tighten First-Fit with Non-Uniform Dimensioning (TFF-NUD), which is documented in Figure 2.

The procedure is quite similar to the TFF-UD method 1. However, some modifications were conducted to obtain a Non-Uniform Wavelength Dimensioning. The modifications can be seen from line 10 to 14 , where the wavelength dimensioning procedure is made, computing the number of wavelengths for each link separately. To do so, we check if all the users passing through link $\ell$ belongs to the set $\mathcal{Q}$ (i.e., if $B P_{c} \leq \beta_{c}$, for all $c \in \mathcal{X}$ ). If any user using link $\ell$ is not on the set $\mathcal{Q}$, then the number of wavelengths on the link is augmented by 1 . Oth${ }_{270}$ erwise, $W_{\ell}$ is the final capacity of link $\ell$, storing it in the set $\mathcal{W}$. Remark that all link inspection is independent, leading to a different amount of wavelengths per link.

The execution of the whole procedure obtains the set of wavelengths on each link, and the set of available wavelengths for each user, executed as $\{\mathcal{W}, \mathcal{U}\}:=$ $\operatorname{TFF}-\mathrm{NUD}(\mathcal{R})$.

Based on the above, we foresee three different kinds of experiments. First, we can evaluate quantitatively the difference in network capacity obtained by computing the wavelengths using the Uniform and Non-Uniform Dimensioning technique, both using a plain First-Fit wavelength allocation procedure. Second, 280 we can contrast the impact of the TQoS policy on saving network capacity, regardless of the dimensioning strategy. Finally, we can examine the importance of considering both strategies together in the dimensioning problem, these are the TQoS policy with the Non-Uniform Dimensioning, and how much each technique affect by itself in saving network capacity. Next, we present some numerical examples to assess these concerns. 


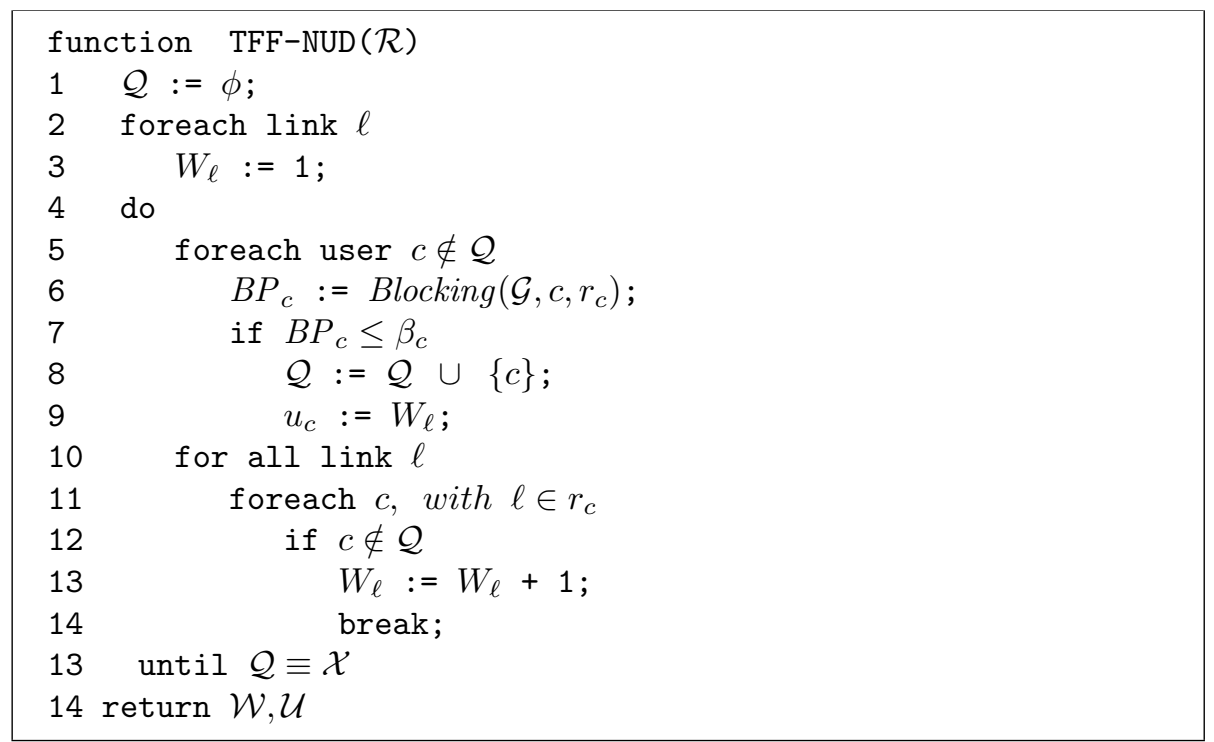

Figure 2: TFF-NUD procedure to compute the number of wavelengths on the network and the amount of wavelengths available to each user using a First-Fit wavelength assignment with a tight quality of service policy with a Non-Uniform Wavelength Dimensioning.

\section{Numerical Results}

As commonly used [8, 27, 17, in this work the total network capacity $C_{n e t}(A)$ is the sum of all wavelengths of all network links computed by method $A$, that is, $C_{n e t}(A)=\sum_{\ell \in \mathcal{L}} W_{\ell}$, where $W_{\ell}$ is the number of wavelengths assigned by $A$ to link $\ell$. This definition is justified because the cost of most components in an optical network is mainly affected by this parameter. In fact, it determines how many infrastructure resources are needed to achieve network operation [28].

As mentioned in the prior section, we adopt the First-Fit technique to solve the wavelength assignment problem in all the numerical examples. In the text, we called it FF. However, recall that the Tighten QoS provision policy (TQoS) alters the First-Fit strategy by limiting the number of wavelengths available to each user, to provide a tighter quality of service policy. In the experiments, we denoted the modified version of the First-Fit strategy as TFF.

To judge the output of the methods under several scenarios, we executed the algorithms on diverse real network topologies, having different size and connectivity (measured by the network degree $d$, defined in Figure 3 caption). Some of the selected topologies and their corresponding parameters $N, L$, and $d$ are shown in Figure 3. For a fair comparison, the considered procedures use the same fixed routing shortest path (Dijkstra's algorithm).

We used the analytic method LIBPE 24 to obtain the corresponding blocking probabilities, because its accuracy was broadly validated employing simulations, and runs orders of magnitude faster than a Montecarlo simulation. This 

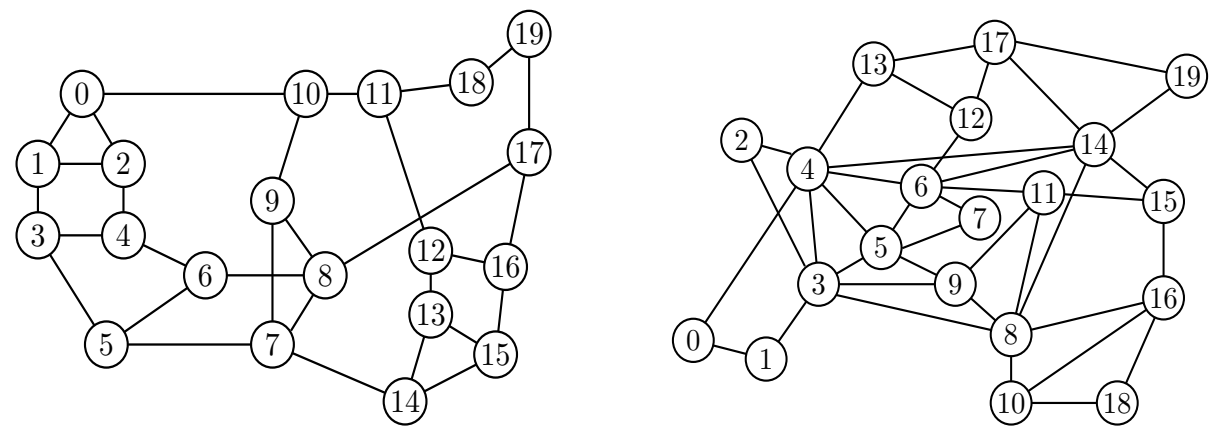

UKNet: 21 nodes, 78 links, $\mathrm{d}=0.19$

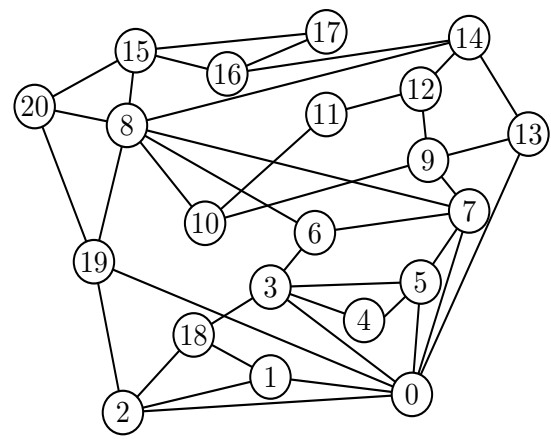

EuroLarge: 43 nodes, 180 links, $\mathrm{d}=0.1$

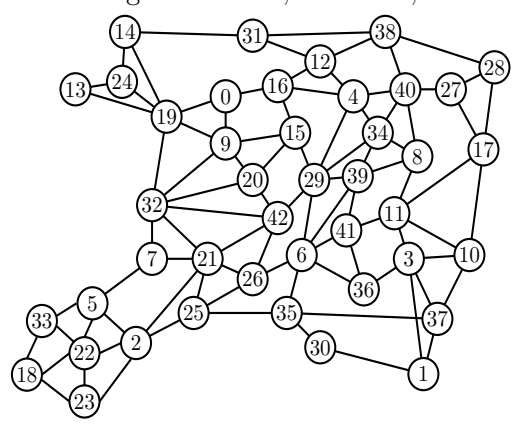

Figure 3: Some of the mesh networks evaluated. The number of links refers to the amount of bi-directional arcs. For instance, the picture shows the EON network topology with 39 edges, which corresponds to 78 arcs. The parameter $d$ is a measure of density: if the graph has $a$ $\operatorname{arcs}$ (twice the number of edges) and $n$ nodes, $d=a /(n(n-1))$.

tool is compatible with our objectives because it can handle the fact that diverse users have a different amount of resources available (wavelengths). We used $\mathrm{HBPE}$ as a module serving all the compared techniques. For supplementary reference for the reader, we executed Montecarlo simulations to cross-validate the results obtained.

To present a robust analysis, we define two different scenarios showing different distributions of the QoS constraints requested by the network users. Remark that some of these scenarios are just examples to analyze different forms of QoS requirements. The idea behind these criteria is to generate QoS scenarios easy to replicate, since allocating the QoS requirements randomly generates scenarios that are not easy to duplicate, and the results may be hard to analyze.

- Homogeneous QoS Requirements (HQoS): The scenario here is the situation where all users have the same threshold equals to $10^{-3}$.

- Heterogeneous QoS Requirements (HeQoS): Though, when a real network operates, it is frequent to find different QoS to different users, 
and the assignments are certainly not arbitrary. Thus, to exemplify, we present a set of experiments which defines each $\beta_{c}$ individually.

We started by choosing a few values to be used as blocking probability thresholds, that we stored in a list $\mathcal{Z}$. In our tests, we adopted the values in the list $\mathcal{Z}=\left[10^{-3}, 10^{-4}, 10^{-5}, 10^{-6}\right]$, sorted in decreasing order. Then, we assigned a value taken in $\mathcal{Z}$ to each user in $\mathcal{X}$. The objective is to obtain different QoS constraints easily to reproduce. For this purpose, we used the length of each user route, measured by its number of hops, to choose a value of $\beta_{c}$ that increases with the length of user c's route.

Explicitly, we classified the users according to the lengths of their routes, say putting all those with the same length $h$ in a set $\mathcal{X}_{h}$, and we assigned to all the users in the same set $\mathcal{X}_{h}$, the same QoS (the same upper-bound of its blocking probability) value. The classification was done as follows. Let $H$ be the length of the longest user path on the network. Then, to all users in set $\mathcal{X}_{h}$ we assign the $z$-th value in $\mathcal{Z}$, computed as $\mathcal{Z}=\left\lceil H \cdot\left|r_{c}\right| /|\mathcal{Z}|\right\rceil$. So, the longer the user path, the stricter the QoS requirement.

In this work, we performed much more experiments than shown in the text.

340 For instance, we explored different heterogeneous QoS constraints scenarios, such as descending QoS constraints (that is, the shorter the user's path, the stricter the QoS required); as well as the case of arbitrary QoS needs (that is a random QoS condition set to each network user). Due to lack of space, we do not show all of them although the TQoS policy and the Non-Uniform capacity mechanism obtains substantial savings in all scenarios.

Hereunder, we will present different sets of experiments and compare the performance of the proposed method with the selected competitor.

\subsection{Uniform $v / s$ Non-Uniform Dimensioning}

To assess the efficiency of both dimensioning methods (UD and NUD) under 350 different situations, we executed the procedures on different network topologies (illustrated in Figure 3 ).

In Figures 4 and 5 we show the network overall capacity computed by the Uniform and Non-Uniform dimensioning method, both using the First-Fit mechanism to allocate the wavelengths. We computed the cases where all users have the same maximum acceptable blocking probability values (Figure 4) and heterogeneous quality of service constraints (Figure 5), as a function of the traffic load.

As expected, to assign a different amount of wavelength to each network link (NUD) obtains significantly better results than Uniform Dimensioning mecha-

360 nism. In fact, NUD obtains on average $23 \%$ fewer wavelengths (for $\varrho=0.3$ ) than needed by the UD strategy, considering both homogeneous and heterogeneous QoS constraint scenarios. It is also worth notice that in each experiment presented here, the same users transmit the same amount of information, having the same corresponding QoS constraints, but thanks to the Non-Uniform 365 Dimensioning technique, the network requires appreciably fewer resources than in the UD case. 
EON, $\beta_{c}=10^{-3}$

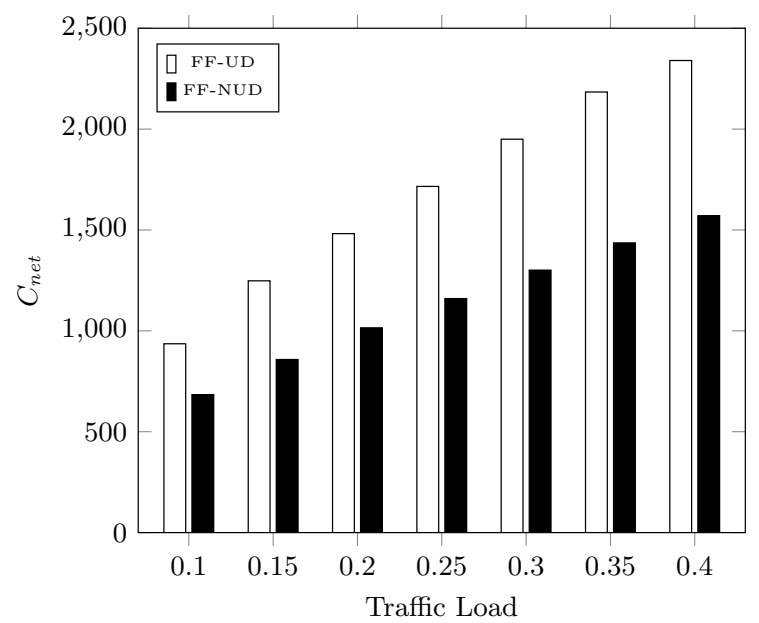

Arpanet, $B=10^{-3}$

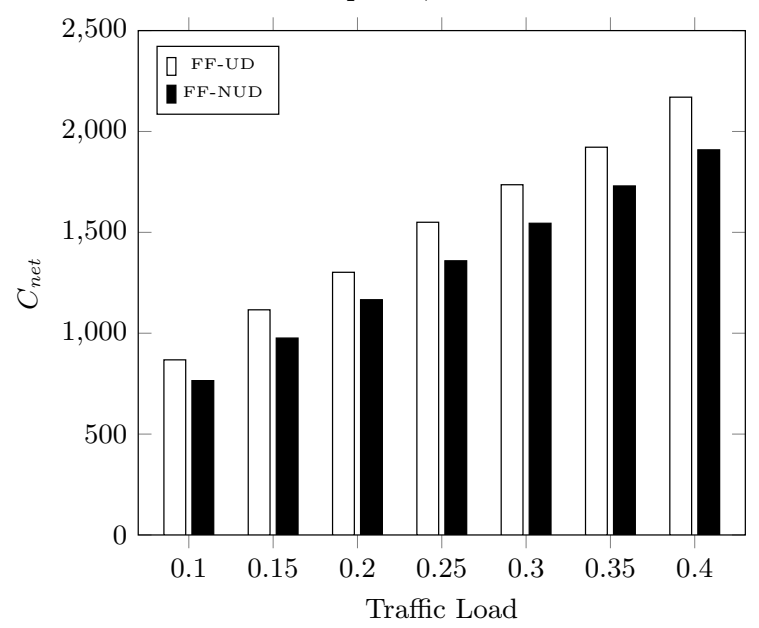

UKNet, $B=10^{-3}$

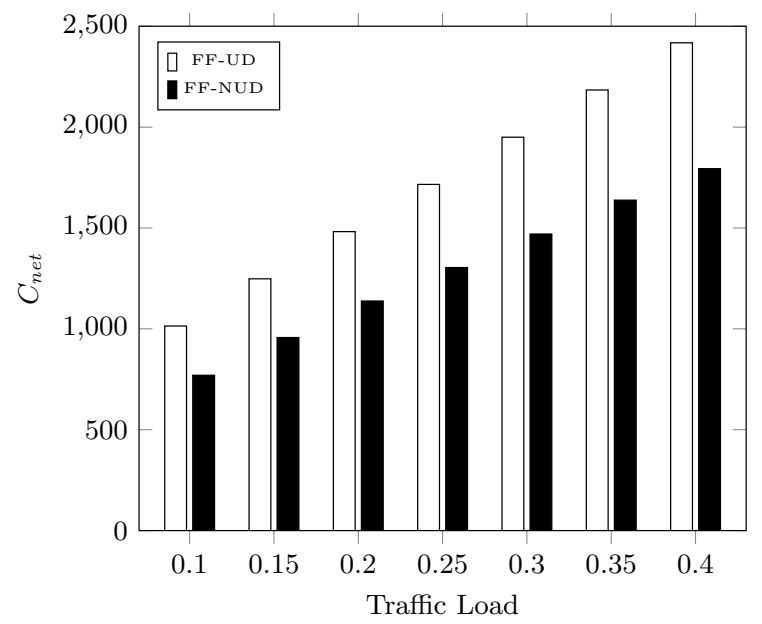

Eurolarge, $B=10^{-3}$

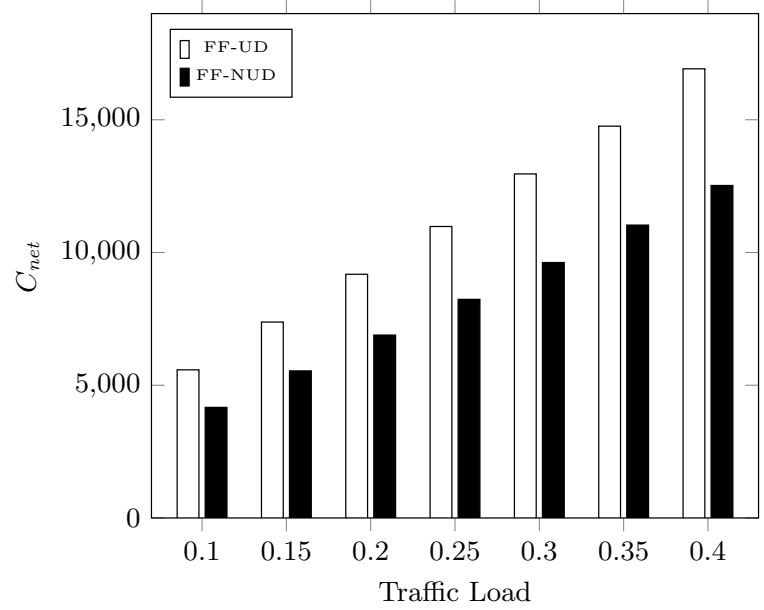

Figure 4: The network total capacity obtained by the Uniform and Non-Uniform Dimensioning with a First-Fit wavelength allocation method (FF-UD and FF-NUD, respectively) on EON, UKNet, Arpanet and Eurolarge real mesh network topologies, for different connection traffic loads, with an homogeneous maximum acceptable blocking probability $\beta_{c}=10^{-3}$.

Remark that even in scenarios with homogeneous QoS constraints (Figure 4) there are significant savings obtained by NUD over UD. This situation occurs due to two factors: the users' paths and network symmetry. First, in this work, we adopted the Shortest-Path strategy to compute the users' path, leading to an unbalanced amount of users on the network links (links with more traffic load demands than others). Under these circumstances, the UD strategy requires more resources than NUD since the dimensioning of the links is determined by 
EON, Ascending $\beta_{c}$

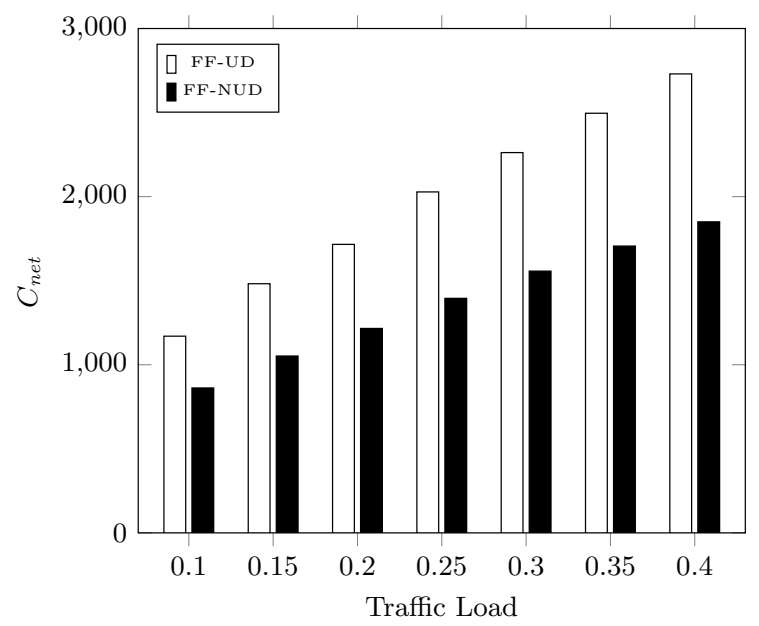

Arpanet, Ascending $\beta_{c}$

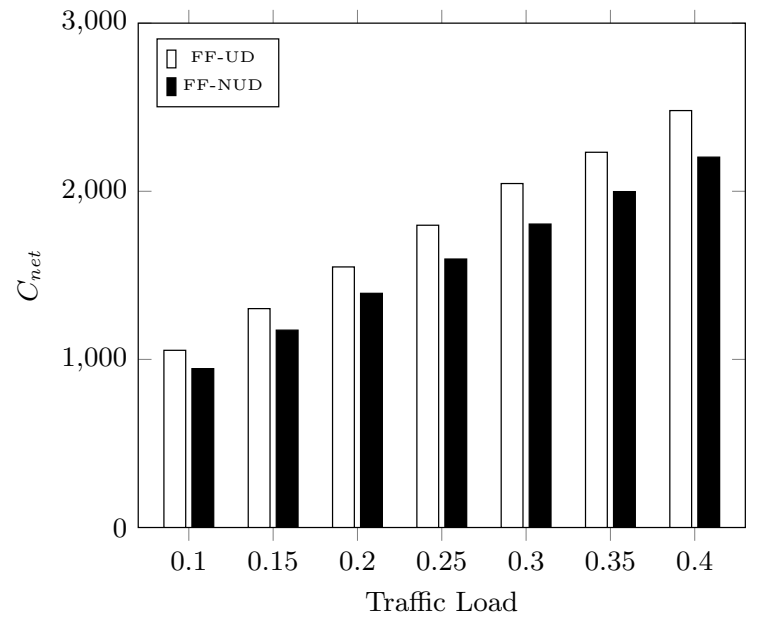

UKNet, Ascending $\beta_{c}$

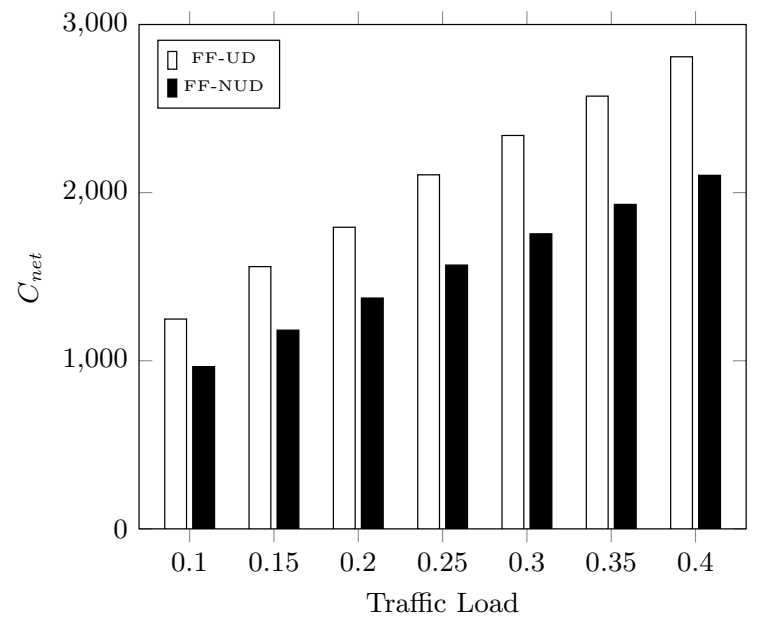

Eurolarge, Ascending $\beta_{c}$

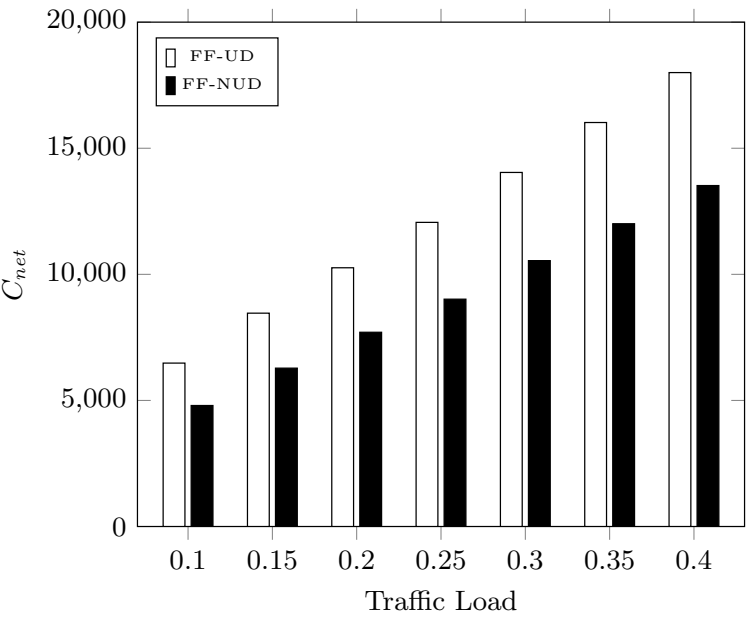

Figure 5: The network total capacity obtained by the Uniform and Non-Uniform Dimensioning, with a First-Fit wavelength allocation method (FF-UD and FF-NUD, respectively) on EON, UKNet, Arpanet and Eurolarge real mesh network topologies, with a heterogeneous maximum acceptable blocking probability $\beta_{c}$. The values of $\beta_{c}$ are chosen between $10^{-3}$ and $10^{-6}$ in ascending order, proportionally to the connections route lengths.

the more loaded link. Second, network topologies are regularly non-symmetric; 375

most of the times, it is not possible to adequately balance the links' traffic loads. This situation benefits the NUD strategy from the capacity saving point of view. 


\subsection{FF $v / s$ Tighten FF}

On the prior set of examples, we analyzed the impact of the dimensioning

380 strategy, both uniform and non-uniform. Now, we discuss the importance of the "Tight QoS" policy. This policy restricts the wavelengths available to each user, offering to each one of them a QoS close to the $\beta_{c}$ value, defined on the service level agreement.

On table 1 we exhibit the network total capacity obtained by TFF and the FF method $\left(C_{n e t}(T F F)\right.$ and $C_{n e t}(F F)$, respectively) for the Uniform Wavelength Dimensioning case. In addition to the foregoing, Table 1 contains the savings obtained by $\mathrm{TFF}$ over plain $\mathrm{FF}$, given in percentage:

$$
\text { Savings }=100 \cdot \frac{C_{n e t}(F F)-C_{n e t}(T F F)}{C_{n e t}(F F)} .
$$

The Savings value is the percentage of capacity savings of TFF over the standard FF procedure. The scenario here is the situation where all users have the same threshold, equals to $10^{-3}$, measured as blocking probability. In general, the conditions of the experiments were the same as the examples in subsection 3.1. but with a Uniform Dimensioning strategy, for the network topologies shown in Figure 3

390 Similarly to Table 1 in Table 2 we show the network overall dimensioning achieved by TFF method compared to the First-Fit one, where all users have different maximum acceptable blocking probability values, following the QoS assignment procedure described in Section 2, as a function of the traffic load, for several network topologies. The experiments were executed using the same wavelength dimensioning procedure (Uniform Dimensioning).

As displayed in both Tables, 1 and 2 in the case of uniform QoS constraints, the TQoS policy improves the FF performance, in terms of overall network capacity computed. For all the scenarios evaluated, TFF requires around $6 \%$ fewer wavelengths than FF method on a Homogeneous QoS constraints scenario, and approximately $7 \%$ fewer wavelengths than FF method on a Heterogeneous QoS constraints case, considering a mean traffic load $\varrho=0.3$, which is a characteristic value [5, 29.

Notice that, in each competition presented here, the objective was to connect the same users with the same QoS requirements (maximum acceptable blocking 405 probability), but thanks to the TQoS policy the network requires fewer resources than FF to do so.

From the tables displayed above, we can see that the savings tend to increase with the topology size. For instance, the smallest topology presented here (the EON network topology) on average, the gain $4.4 \%$ and $4.28 \%$ is obtained in both scenarios presented; meanwhile, on the largest one, Eurolarge network, TFF achieves on average an $8.3 \%$ and $7.94 \%$ of savings, on the same scenarios. When the QoS requirements are the same to all users (Table 1), the longest routes on the network are the hardest one to ensure their QoS constraints; therefore, these routes are the ones that mainly define the network capacity considering a homogeneous dimensioning strategy. Also, note that bigger networks have a 
Table 1: Network capacity achieved by the methods TFF and FF and the Savings obtained, with homogeneous QoS constraints equals to $10^{-3}$, and uniform wavelength dimensioning, for different network topologies and traffic load.

\begin{tabular}{|c|c|c|c|c|c|}
\hline Network Topology & Value & $\bar{\varrho}=0.1$ & $\bar{\varrho}=0.2$ & $\bar{\varrho}=0.3$ & $\bar{\varrho}=0.4$ \\
\hline \multirow{4}{*}{ EON } & $C_{\text {net }}(F F)$ & 936 & 1482 & 1950 & 2340 \\
\cline { 2 - 6 } & $C_{\text {net }}(T F F)$ & 936 & 1404 & 1794 & 2262 \\
\cline { 2 - 6 } & Savings $(\%)$ & 0 & 5.26 & 8.00 & 3.33 \\
\hline \multirow{4}{*}{ UKNet } & $C_{\text {net }}(F F)$ & 1014 & 1482 & 1950 & 2418 \\
\cline { 2 - 6 } & $C_{\text {net }}(T F F)$ & 936 & 1482 & 1872 & 2262 \\
\cline { 2 - 6 } Arpanet & Savings $(\%)$ & 7.69 & 0 & 4.00 & 6.45 \\
\hline \multirow{5}{*}{ Eurolarge } & $C_{\text {net }}(F F)$ & 868 & 1302 & 1736 & 2170 \\
\cline { 2 - 6 } & $C_{\text {net }}(T F F)$ & 806 & 1240 & 1674 & 2046 \\
\cline { 2 - 6 } & Savings $(\%)$ & 7.14 & 4.76 & 3.57 & 5.71 \\
\hline & $C_{n e t}(F F)$ & 5580 & 9180 & 12960 & 1690 \\
\cline { 2 - 6 } & $C_{\text {net }}(T F F)$ & 5040 & 8460 & 11700 & 15480 \\
\cline { 2 - 6 } & Savings $(\%)$ & 9.67 & 7.84 & 9.72 & 8.51 \\
\hline
\end{tabular}

wider variety of path lengths. Hence, when stretching the QoS provision, the savings obtained grow when the network size increases. These circumstances can also be seen on the heterogeneous QoS constraints scenarios (see Table 2).

Despite TFF savings achieved in almost all the scenarios, there is not an evident logic behavior between the savings obtained and the mean users' traffic load. For instance, in Table 1 the UKNet savings are $7.69 \%, 0 \%$ and $4 \%$ with a $\varrho$ equals to $0.1,0.2$ and 0.3 respectively. This phenomenon can be explained because the homogeneous dimensioning scheme increases in discrete values since, at a certain point, a little increase in the traffic load causes that all the links capacity must increase together by one.

\subsection{TQoS policy and Non-Uniform Dimensioning}

On the preceding examples, both dimensioning decisions were assessed separately, these are the "Tight QoS" policy, and to assign a Non-Uniform capacity to the network links. Now, we discuss how much both strategies together (TFF430 NUD) impact the network performance in comparison to the commonly used approach, known as First-Fit plus a Uniform Dimensioning (FF-UD) [8, 13, 11.

As in the preceding subsections, in Figures, 6 and 7, we illustrate the network total capacity achieved by TFF-NUD method in contrast with the FF-UD 
Table 2: Network capacity achieved by the methods TFF and FF and the Savings obtained, with heterogeneous QoS constraints and uniform wavelength dimensioning, for different network topologies and traffic load.

\begin{tabular}{|c|c|c|c|c|c|}
\hline Network Topology & Value & $\bar{\varrho}=0.1$ & $\bar{\varrho}=0.2$ & $\bar{\varrho}=0.3$ & $\bar{\varrho}=0.4$ \\
\hline \multirow{4}{*}{ EON } & $C_{\text {net }}(F F)$ & 1170 & 1716 & 2262 & 2730 \\
\cline { 2 - 6 } & $C_{\text {net }}(T F F)$ & 1170 & 1638 & 2106 & 2574 \\
\cline { 2 - 6 } & Savings $(\%)$ & 0 & 4.54 & 6.89 & 5.71 \\
\hline \multirow{4}{*}{ UKNet } & $C_{\text {net }}(F F)$ & 1248 & 1794 & 2340 & 2808 \\
\cline { 2 - 6 } & $C_{\text {net }}(T F F)$ & 1170 & 1716 & 2184 & 2652 \\
\cline { 2 - 6 } Arpanet & Savings $(\%)$ & 6.25 & 4.34 & 6.66 & 5.55 \\
\hline \multirow{5}{*}{ Eurolarge } & $C_{n e t}(F F)$ & 1054 & 1550 & 2046 & 2480 \\
\cline { 2 - 6 } & $C_{\text {net }}(T F F)$ & 992 & 1488 & 1922 & 2356 \\
\cline { 2 - 6 } & Savings $(\%)$ & 5.88 & 4.00 & 6.06 & 5.00 \\
\hline & $C_{n e t}(F F)$ & 6480 & 10260 & 14040 & 18000 \\
\cline { 2 - 6 } & $C_{\text {net }}(T F F)$ & 5940 & 9360 & 12960 & 16740 \\
\cline { 2 - 6 } & Savings $(\%)$ & 8.33 & 8.77 & 7.69 & 7.00 \\
\hline
\end{tabular}

one. We obtained these metrics in the scenario where all users have a Quality of service threshold (measured as user blocking probability) following the Homogeneous QoS assignment procedure explained earlier (Figures 6), and the Heterogeneous QoS assignment mechanism (Figures 7), as a function of the traffic load and for several network topologies.

Remark that each planning decision (non-uniform dimensioning strategy and 440 tight QoS provision) obtain savings separately, but when they are used together, the savings are near to $30 \%$ in comparison to the conventional approach (FFUD). On the numerical examples, we can appreciate that the Non-Uniform capacity decision obtains more savings than the TQoS policy. In fact, the NonUniform dimensioning mechanism achieves approximately $80 \%$ of the savings (the offered QoS tightening policy procures the remaining 20\%).

We believe essential to notice that both strategies analyzed here can be executed before the network operation. Then, the network operation relies only on routing tables, holding the information needed by the network to operate. For instance, to implement the TQoS policy, it is only necessary to store each user 450 path and its maximum available wavelength, so during the operation of the network, the First-fit wavelength search is executed only on the available capacity defined to the user. This is important because neither strategy proposed here 
EON, $\beta_{c}=10^{-3}$

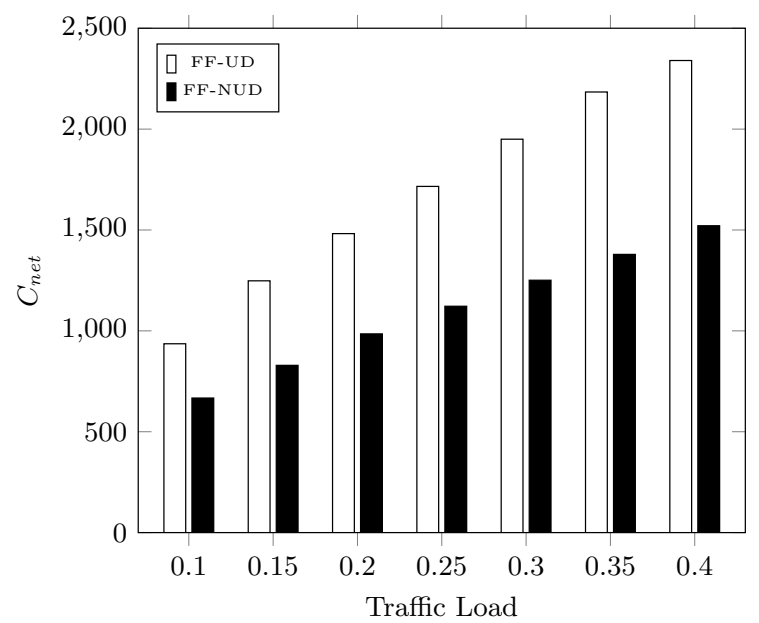

Arpanet, $B=10^{-3}$

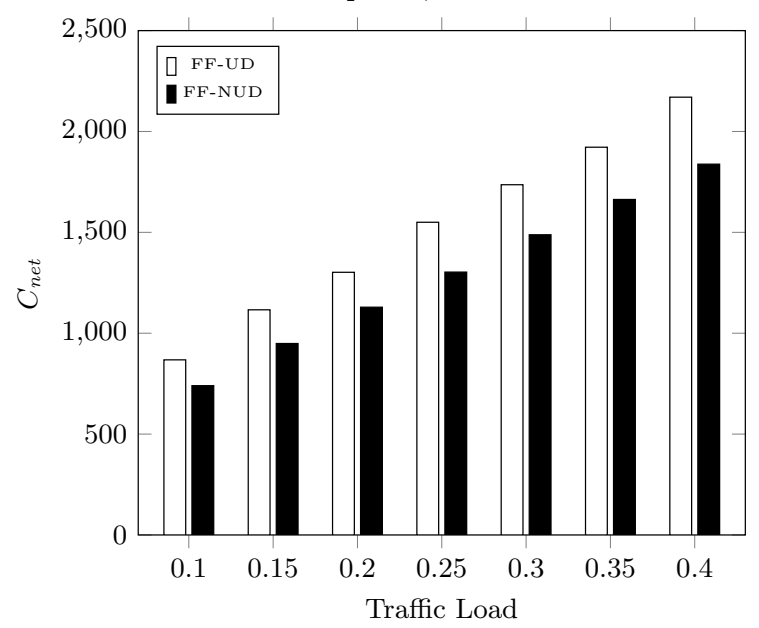

UKNet, $B=10^{-3}$

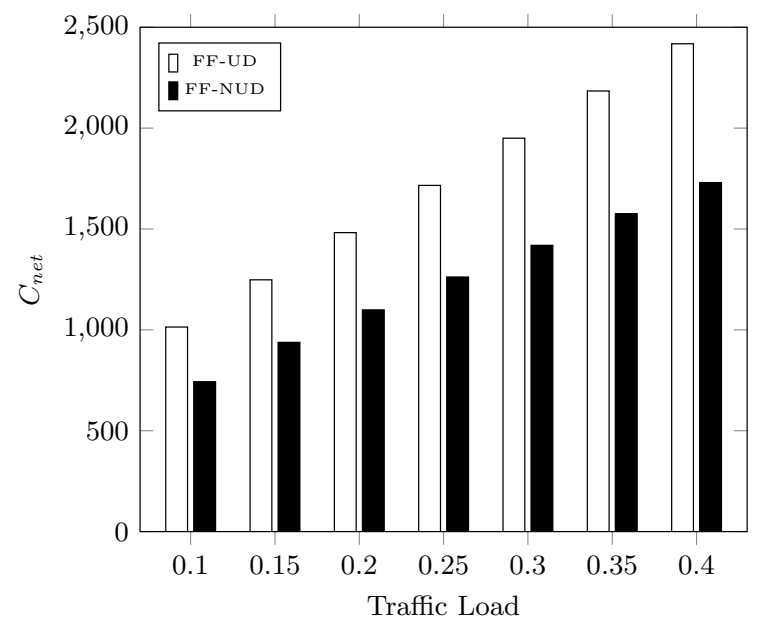

Eurolarge, $B=10^{-3}$

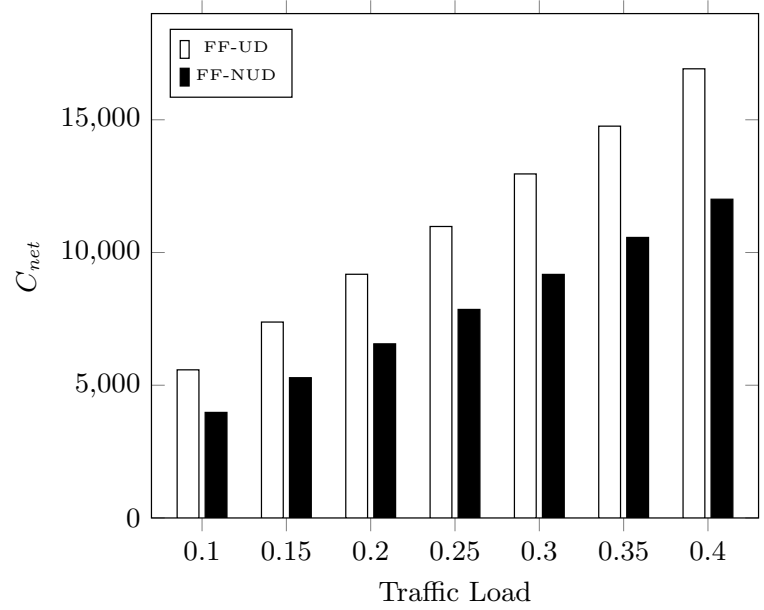

Figure 6: The network total capacity obtained by the TFF-NUD and FF-UD method on EON, UKNet, Arpanet and Eurolarge real mesh network topologies, for different connection traffic loads, with an homogeneous maximum acceptable blocking probability $\beta_{c}=10^{-3}$.

generates an extra overhead to the network operation.

\section{Conclusions}

455 To the best of our understanding, there is not an analysis in the literature examining the consequences of taking different wavelength dimensioning approaches, or how much tightening the difference between the offered quality of service and the one requested on the SLA impacts the network capacity in 
EON, Ascending $\beta_{c}$

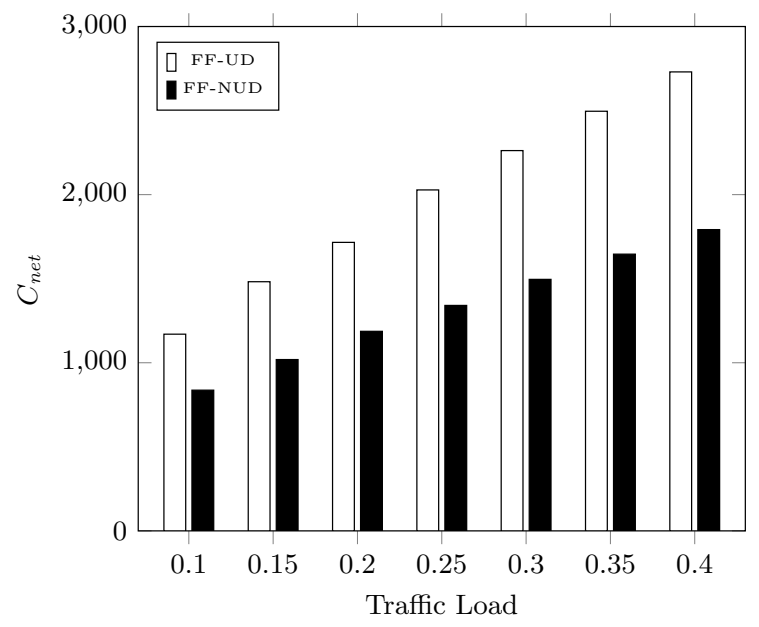

Arpanet, Ascending $\beta_{c}$

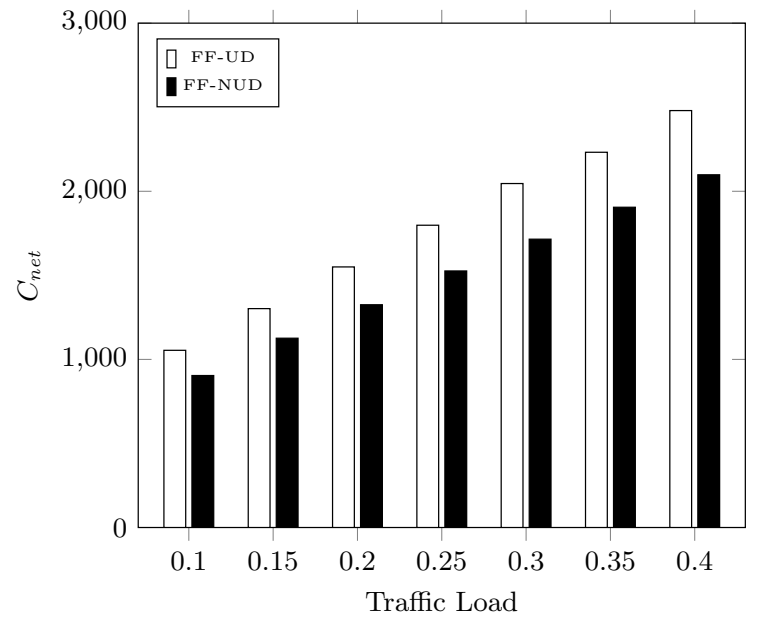

UKNet, Ascending $\beta_{c}$

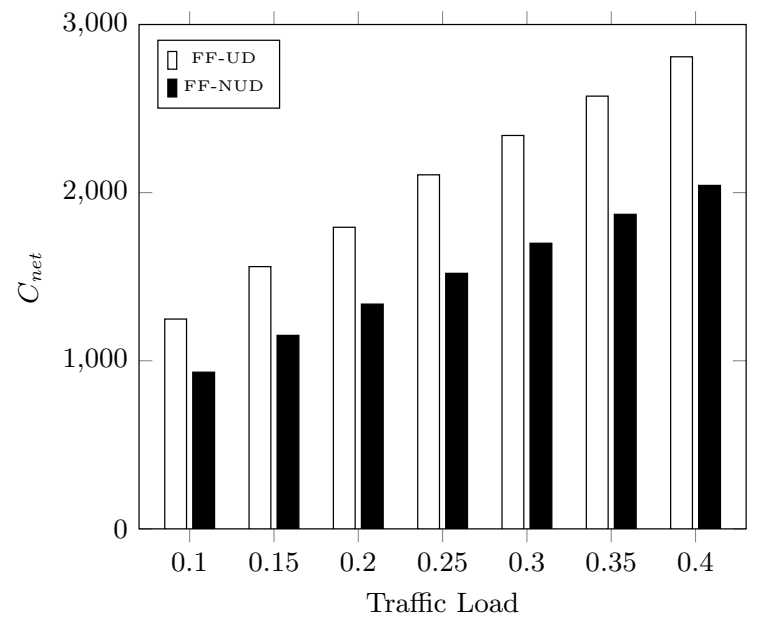

Eurolarge, Ascending $\beta_{c}$

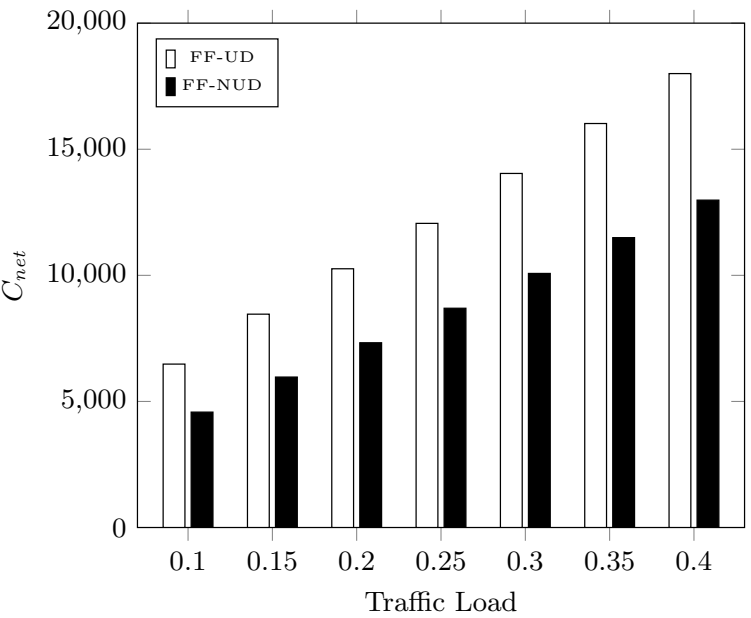

Figure 7: The network total capacity obtained by the TFF-NUD and FF-UD method on EON, UKNet, Arpanet and Eurolarge real mesh network topologies, for different connection traffic loads, with an heterogeneous maximum acceptable blocking probability $\beta_{c}$. The values of $\beta_{c}$ are chosen between $10^{-3}$ and $10^{-6}$ in an ascending order, proportionally to the connections route lengths.

optical networks with wavelength continuity constraints. We believe that these 460 issues are relevant to be taking into account, mainly because diverse strategies on both concerns may affect the overall network capacity dimensioning. Even more, with the impending capacity crunch scenario on fiber cable communications. Consequently, we assessed the impact of both network planning decisions.

One strategy is to dimension a different capacity to each network link since 

ening the gap between the offered and the requested quality of service for each user. To achieve the latter, we restrict the wavelengths available to each user (Wavelength Grouping), thus offering a low difference between the offered quality of service and the quality of service stated on the Service Level Agreement.

As exhibited in the numerical experiments, both planning decisions obtain notorious savings on the overall network capacity, notwithstanding of the scenarios appraised. Furthermore, the online network operation is fast and straightforward, since both strategies are executed prior the network operation, and the TQoS policy relies on the same routing tables used to store the users' path by adding the wavelength available per user.

As a final remark, Elastic Optical Networks (EON) [30, 4, 31 are an essential and current topic to address. However, the analysis presented here does not apply to the EON context. This is because the fast evaluation of the users' blocking probability is mandatory to compute the dimensioning strategies presented here, and to the best of our knowledge, there is not an accurate and fast mathematical method to calculate the blocking probability in EON (the LIBPE method is only valid in non-elastic optical networks with wavelength continuity constraints). In future work, we will assess the impact of the strategies presented in this text on the flexible optical network architectures.

\section{Acknowledgments}

This work received financial support from FONDEF ID14I20129 and STICAMSUD 19STIC-01, CONICYT, Chile . These projects are then gratefully acknowledged. Additionally, we thank Dr. Ricardo Olivares from UTFSM, and the anonymous referees for their useful suggestions.

\section{Appendix A. QoS Evaluation}

Hereunder, we summarize the analytic evaluation of the users blocking probability taking into account the wavelength continuity constraints, denoted as "Layered Iterative Blocking Probability Evaluation" (LIBPE). The approximate solution follows a 4 stage strategy, explained next. However, full details can be

Network Division. From the vocabulary point of view, a network $\mathcal{G}$ with capacity $W$ on each link is composed of $W$ networks or "layers" operating in tandem, $\left\langle\mathcal{G}_{1}, \mathcal{G}_{2}, \ldots, \mathcal{G}_{W}\right\rangle$, with the same topology as the original one, but where each link has a capacity equal to 1 . Then, on a First-Fit scheme, an arriving user 500 will look for room in layer 1 first, if this fails, in $\mathcal{G}_{2}$, and so on, until it finds available capacity in one of the $W$ layers, or until all of them block it. Note that, if the capacities of the network links are different, the layers $\mathcal{G}_{w}$ are then a subset of $\mathcal{G}$ where the links with less capacity than $w$ are deleted (e.g., in a network with capacity equals to 2 on each link but one having 1 wavelength 


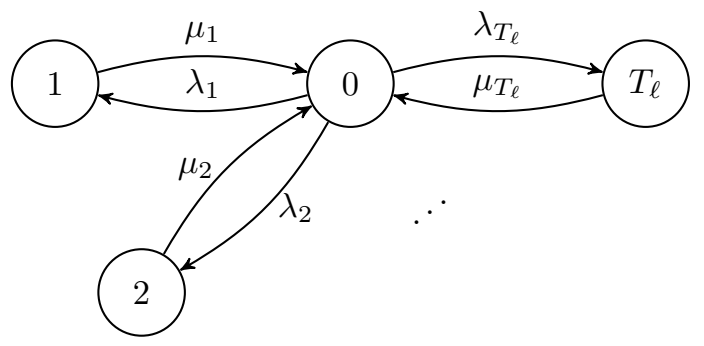

Figure A.8: Markov chain modeling the use of a given link in a network where all links have a single wavelength. There are $T_{\ell}$ connections using the link. State $c$ means that user $c$ is using the link, $c=1,2, \ldots, T_{\ell}$. State 0 means that the only wavelength of the link is available. Arrival rate of a communication request of user $c: \lambda_{c}=1 / t_{O F F_{c}}$. Service rate (by the link) of a transmission of user $c: \mu_{c}=1 / t_{O N c}$.

available, in the network division procedure the first layer $\mathcal{G}_{1}$ is constituted by all the links, and the second layer $\mathcal{G}_{2}$ possess all the links available except the link with a single wavelength).

LIBPE will then follow a decomposition approach: we analyze each layer in isolation, but its parameters will depend on what happens on the other layers. Next, we present a dependency model between the mean lengths of the OFF periods to take into account the interaction between the $W$ networks.

Analytical model when $W=1$. Since the network is divided into a sequence of $W$ networks/layers, where each link has a single wavelength, then let us solve the case of $W=1$. Choose one link in the network, say link $\ell$. Some users (at least one) use this link in their routes, some do not. Hence, denote by $T_{\ell}$ the number of users using $\ell$, and renumber the users so that those using link $\ell$ are $1,2, \ldots, T_{\ell}$.

Assume the system is in equilibrium. Let $B L_{c, \ell}$ be the blocking probability of user $c$ at link $\ell$, that is, the probability that user $c$ connection request arriving 520 at link $\ell$ finds it busy. We assume Markovian conditions, that is, exponentially distributed arrivals and service times, with respective rates $\lambda_{c}=1 / t_{O F F_{c}}$ and $\mu_{c}=1 / t_{O N_{c}}$. The continuous time stochastic process $Z=\{Z(t), t \geq 0\}$ on the state space $\left\{0,1,2, \ldots, T_{\ell}\right\}$, representing the link state at time $t$ is then Markov (see Figure A.8). A straightforward analysis of this Markov chain immediately leads to

$$
\pi_{c}=\frac{\phi_{c}}{1+\phi}, \quad c=1,2, \ldots, T_{\ell}, \quad \pi_{0}=\frac{1}{1+\phi},
$$

where $\phi_{c}$ is the ratio $\phi_{c}=\lambda_{c} / \mu_{c}=t_{O N_{c}} / t_{O F_{c}}$ and $\phi$ is the sum $\phi=\phi_{1}+\cdots+$ $\phi_{T_{\ell}}$.

The blocking probability $B L_{c, \ell}$ is the ratio between the probability of a user $c$ request being blocked for lack of resources and the probability of all possible scenarios when user $c$ wants to transmit. It can also be derived marking user $c$ 
arrivals and analyzing the chain embedded at the marked transition epochs. Then, according to A.1 the result is

$$
B L_{c, \ell}=\frac{1-\pi_{0}-\pi_{c}}{1-\pi_{c}}=\frac{\phi-\phi_{c}}{1+\phi-\phi_{c}} .
$$

As we are assessing the blocking probability on any $\mathcal{G}_{w}$ networks, we can resolve that each link blocking probability on the w-th network $B L_{c, \ell}^{w}$ is equal to $t_{O N}$ and $t_{O F F}$.

Then, the user blocking probability $c$, with $c \in \mathcal{X}$, on network $\mathcal{G}_{w}$, can be approximated by means of the usual link independence assumption [32, 33, 34, [35. This is

$$
B C_{c}^{w}=1-\prod_{\ell \in r_{c}}\left(1-B L_{c, \ell}^{w}\right) .
$$
tive circumstances, where many users can often be trying to access simultaneously the same resources. Thus, to improve the quality of the solution, a fixed point method proposed by Kelly [36] is then used.

Network interaction. Now, we need to take into account the interaction between each of the $W$ networks $\mathcal{G}_{1}, \ldots, \mathcal{G}_{W}$. We seize this interaction iteratively updating the values characterizing the ON-OFF arrival processes of all the network users on each layer $\mathcal{G}_{w}$. For this purpose, let us denote $t_{O N_{c, w}}$ and $t_{O F F_{c, w}}$ as the average values of $\mathrm{ON}$ and $\mathrm{OFF}$ periods for user $c$ in network $\mathcal{G}_{w}$. About $t_{O N_{c, w}}$, is the time used by source $c$ to transmit because this period is the same 550 regardless the network used to transmit, then the parameter $t_{O N_{c, w}}$, is equal to $t_{O N_{c}}$ for all $w$. This situation means that the dependencies between layers is captured only by the $t_{O F F_{c, w}}$ values.

The core of our scheme focuses then on those dependencies between layers, which are of three types as follows:

- Sequential dependency: When a request from user $c$ is accepted at layer $\mathcal{G}_{w}$, the next layers will not receive it. In other words, a wavelength in the sequence receives a request from user $c$ only if it is blocked in every previous wavelengths in the sequence. Therefore, to represent the mentioned situation, the $t_{O F F_{c, w}}$ values will grow by the quantity $t_{O F F_{c}}+$ $t_{O N_{c}}$ (from now on denoted as $\tau_{c}$ ) in every $w^{\prime}>1$, for each request transmitted on the wavelengths previous to $w^{\prime}$.

- Backward dependency: After user $c$ is blocked on network $\mathcal{G}_{1}$ and accepted in any of the next wavelengths, the next transmission request (in $\mathcal{G}_{1}$ ) of user $c$ will happen after one transmission period -because the first request was accepted- and one idle period (mean length $t_{O F F_{c}}$ ) to recollect new data to transmit. Therefore, all blocked users in network $\mathcal{G}_{1}$, but accepted on any of the next networks $\mathcal{G}_{w}, w>1$, make $t_{O F F_{c, 1}}$ increase 
by $\tau_{c}$. Remark that this $t_{O F F_{c, 1}}$ growth does not influence only on the first layer $\mathcal{G}_{1}$, but every layer. Although, it is sufficient to consider this dependency only on $\mathcal{G}_{1}$ because the sequential dependency will spread this effect.

- General blocking dependency: This considers the scenario when a user $c$ request is blocked on every wavelength (every network $\mathcal{G}_{w}$, for all $w$ ). In this case, user $c$ start again to recollect new data to transmit (using one OFF period). Therefore, all blocked users in the final network $\mathcal{G}_{W}$ make the $t_{O F F_{c, 1}}$ value increase by $t_{O F F_{c}}$.

Respecting the dependencies just introduced, we propose two equations. For this purpose, we denote $B C_{c}^{w}$ the blocking probability of user $c$ at layer $\mathcal{G}_{w}$.

- First Wavelength $(w=1)$. On the first wavelength, that is, on the first layer $\mathcal{G}_{1}$, we take into account the last 2 dependencies. Then:

$$
t_{O F F_{c, 1}}=t_{O F F_{c}}+\tau_{c} B C_{c}^{1}-t_{O N c} \prod_{k=1}^{W} B C_{c}^{k} .
$$

- Next wavelengths now. Observe that the next layers only take into account the sequential dependency. Then, the mean length of the OFF period corresponding to user $c$ for wavelength $w>1$ is

$$
t_{O F F_{c, w}}=t_{O F F_{c, w-1}}+\tau_{c} \sum_{m=1}^{w-1}\left(\frac{1}{B C_{c}^{m}}-1\right) .
$$

The sum on the Equation A.5 represents the mean number of times that the user transmission is accepted on previous networks, i.e., $m \leq w$ until the request to transmit is blocked (Geometric distribution analysis).

Overall User Blocking Evaluation. Finally, taking into account all the layers, the overall blocking probability of the user $c$ is computed as:

$$
B C_{c}=\prod_{\text {all } w} B C_{c}^{w} .
$$

Remark that, to take into account the wavelength grouping policy, the equation $($ A.6 $)$ is executed only on the user $c$ available wavelengths.

\section{References}

[1] A. R. Chraplyvy, Plenary paper: The coming capacity crunch, in: 2009 35th European Conference on Optical Communication, 2009, p. 8007.

[2] A. D. Ellis, N. M. Suibhne, D. Saad, D. N. Payne, Communication networks beyond the capacity crunch, Philosophical Transactions of the Royal Society A: Mathematical, Physical and Engineering Sciences 374 (2062) (2016) 20150191. 
[10] L. Tan, Q. Yang, J. Ma, S. Jiang, Wavelength Dimensioning of Optical Transport Networks Over Nongeosychronous Satellite Constellations, Journal of Optical Communications and Networking 2 (4) (2010) 166-174.

[11] R. T. Koganti, D. Sidhu, Analysis of routing and wavelength assignment in large WDM networks, in: Procedia Computer Science, Vol. 34, 2014, pp. 71-78.

[12] B. Mukherjee, Optical WDM networks, Vol. 26, Springer Science \& Business Media, 2006.

[13] B. C. Chatterjee, N. Sarma, P. Pratim Sahu, Review and Performance Analysis on Routing and Wavelength Assignment Approaches for Optical Networks., IETE Technical Review 30 (1) (2013) 12-23. 
[14] W. Liao, C. H. Loi, Providing service differentiation for optical-burstswitched networks, Journal of Lightwave Technology 22 (7) (2004) 16511660.

[15] D. H. Hailu, G. G. Lema, E. A. Yekun, S. H. Kebede, Unified study of Quality of Service (QoS) in OPS/OBS networks, Optical Fiber Technology 36 (2017) 394-402. doi:10.1016/J. YOFTE. 2017.05.016

[16] S. Mohd Sam, S. Mohd Daud, K. Kamardin, N. Maarop, Study of Qos Performance in Optical Burst Switched Networks (OBS), Indian Journal of Science and Technology 9 (48). doi:10.17485/ijst/2016/v9i48/99269.

[17] N. Jara, R. Vallejos, G. Rubino, A method for joint routing, wavelength dimensioning and fault tolerance for any set of simultaneous failures on dynamic WDM optical networks, Optical Fiber Technology 38 (2017) 30-40. doi:10.1016/j.yofte.2017.08.001.

n URL http://www.sciencedirect.com/science/article/pii/ S1068520017301712

[18] F. Z. Khan, M. F. Hayat, T. Holynski, M. J. Khan, Towards dynamic wavelength grouping for QoS in optical burst-switched networks, in: 2017 40th International Conference on Telecommunications and Signal Processing (TSP), IEEE, 2017, pp. 79-85. doi:10.1109/TSP.2017.8075941. URL http://ieeexplore.ieee.org/document/8075941/

[19] M. A. Aydin, T. Atmaca, O. C. Turna, H. Zaim, T. Eido, Regulative Wavelength Grouping Approach for QoS in OBS Networks, in: 2009 Fifth Advanced International Conference on Telecommunications, IEEE, 2009, pp. 254-260. doi:10.1109/AICT.2009.50. URL http://ieeexplore.ieee.org/document/5072441/

[20] Q. Zhang, V. M. Vokkarane, J. P. Jue, B. Chen, Absolute QoS differentiation in optical burst-switched networks, IEEE Journal on Selected Areas in Communications 22 (9) (2004) 1781-1795.

[21] E. W. Dijkstra, A note on two problems in connection with graphs, Numerische Mathematik 1 (1) (1959) 269-271.

[22] A. Zapata-Beghelli, P. Bayvel, Dynamic Versus Static Wavelength-Routed Optical Networks, Lightwave Technology, Journal of 26 (20) (2008) 34033415 .

[23] A. Mokhtar, M. Azizo\uglu, Adaptive Wavelength Routing in All-optical Networks, IEEE/ACM Trans. Netw. 6 (2) (1998) 197-206. doi:10.1109/ 90.664268 .

URL http://dx.doi.org/10.1109/90.664268

[24] N. Jara, R. Vallejos, G. Rubino, Blocking Evaluation and Wavelength Dimensioning of Dynamic WDM Networks Without Wavelength Conversion, Journal of Optical Communications and Networking 9 (8) (2017) 625. 
[25] F. Shirin Abkenar, A. Ghaffarpour Rahbar, Study and Analysis of Routing and Spectrum Allocation (RSA) and Routing, Modulation and Spectrum Allocation (RMSA) Algorithms in Elastic Optical Networks (EONs) (2017). arXiv:9780201398298, doi:10.1016/j.osn.2016.08.003

[26] N. Jara, R. Vallejos, G. Rubino, Blocking evaluation of dynamic WDM networks without wavelength conversion, in: 2016 21st European Conference on Networks and Optical Communications (NOC), 2016, pp. 141-146.

[27] A. N. Khan, An improved approximate network blocking probability model for all-optical WDM Networks with heterogeneous link capacities, Optical Fiber Technology 38 (2017) 7-16. doi:10.1016/J.YOFTE.2017.07.008.

[28] D. Banerjee, B. Mukherjee, Wavelength-routed optical networks: Linear formulation, resource budgeting tradeoffs, and a reconfiguration study, IEEE/ACM Transactions on Networking 8 (5) (2000) 598-607.

[29] R. Bolla, R. Bruschi, A. Cianfrani, M. Listanti, Enabling backbone networks to sleep, IEEE Network 25 (2) (2011) 26-31. doi:10.1109/MNET. 2011.5730525 URL http://ieeexplore.ieee.org/document/5730525/

[30] P. Layec, A. Morea, F. Vacondio, O. Rival, J.-C. Antona, Elastic I optical networks: The global evolution to software configurable optical networks, Bell Labs Technical Journal 18 (3) (2013) 133-151. doi:10.1002/bltj.21631. URL http://ieeexplore.ieee.org/lpdocs/epic03/wrapper.htm? arnumber $=6772713$

[31] K. Christodoulopoulos, I. Tomkos, E. A. Varvarigos, Elastic bandwidth allocation in flexible ofdm-based optical networks, Journal of Lightwave Technology 29 (9) (2011) 1354-1366.

[32] A. Alyatama, Computing blocking probabilities in survivable WDM optical networks, Photonic Network Communications 27 (1) (2014) 34-46.

[33] J. Triay, C. Cervelló-Pastor, V. M. Vokkarane, Analytical Blocking Probability Model for Hybrid Immediate and Advance Reservations in Optical WDM Networks, IEEE/ACM Trans. Netw. 21 (6) (2013) 1890-1903. doi:10.1109/TNET.2012.2235857. URL http://dx.doi.org/10.1109/TNET.2012.2235857

[34] Y. Zhao, J. Zhang, Blocking probability analysis model for flexible spectrum optical networks, Chin. Opt. Lett. 12 (7) (2014) 70601. URL http://col.osa.org/abstract.cfm?URI=col-12-7-070601

[35] L. H. Bonani, I. E. Fonseca, Estimating the blocking probability in 710 wavelength-routed optical networks, Optical Switching and Networking 10 (4) (2013) 430-438. doi:http://dx.doi.org/10.1016/j.osn.2013. 
07.001

URL

http://www.sciencedirect.com/science/article/pii/ S1573427713000489

715 [36] F. P. Kelly, Loss Networks, The Annals of Applied Probability 1 (3) (1991) pp. 319-378. 